\title{
A Novel Approach of IoT Services Orchestration Based on Multiple Sensor and Actuator Platforms Using Virtual Objects in Online IoT App-Store
}

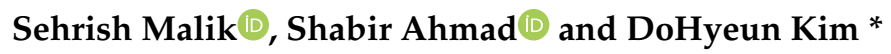 \\ Computer Engineering Department, Jeju National University, Jeju-si 63243, Korea; serrym29@gmail.com (S.M.); \\ shabir@jejunu.ac.kr (S.A.) \\ * Correspondence: kimdh@jejunu.ac.kr; Tel.: +82-64-754-3658
}

Received: 8 October 2019; Accepted: 21 October 2019; Published: 22 October 2019

check for updates

\begin{abstract}
The information and communication technology (ICT) is witnessing a revolutionary era with the advancements in the Internet of Things (IoT). An IoT network is a combination of sensor and actuator networks, connected and communicating in certain ways to design and provide IoT services to the end users. These IoT services are created by mapping physical-world objects into virtual-world objects. In this work, we propose a novel approach of IoT services orchestration based on multiple sensor and actuator platforms using virtual objects in online IoT application-store (app-store). In this work, we focused on combining the concepts of do-it-yourself (DIY) IoT marketplace, virtual objects (VOs), and virtual services. We built a fusion IoT services platform on a previously proposed IoT application store. The IoT application store enables the sharing and discovery of IoT VOs, along with micro-services associated with each VO uploaded into the application store. The fusion IoT services platform enables the user to fetch the desired or all VOs from the IoT app store and map the available VOs to form the fusion IoT services. The user can either select all the available VOs and see all the possible services' combinations or select the desired (DIY) services and customize the virtual services scope. The performance of the proposed fusion IoT services platform was evaluated on the basis of the service connection times, service response times with varying load of VOs, virtual users, and active platforms. The proposed idea also offers a sustainable solution by proposing the reuse of existing resources and reducing duplicate deployments, which can lessen the total cost of the physical networks' deployment and maintenance. To the best of our knowledge, the proposed work is the first of its kind.
\end{abstract}

Keywords: IoT application store; virtual objects (VOs); virtual services; sensor platform; actuator platform

\section{Introduction}

The information and communication technology (ICT) is witnessing a revolutionary era with the advancements in the Internet of Things (IoT). These advancements are resulting in a huge deployment of sensors, actuators, RFID (radio-frequency identification) tags and mobile equipment in order to convert the world around us into a smart world [1-3]. IoT means in fact the combination of these sensing and actuating devices which bear different processing capabilities and are connected to form an IoT network. An IoT network is a combination of sensor and actuator networks that are connected and communicate in certain ways to design and provide IoT services to the users. These IoT services are created by mapping physical-world objects into virtual-world objects, using the collected real-world data and controlling the real-world objects. The virtual world is composed of virtual objects (VOs). Virtual objects are the digital counterparts of physical objects. A physical object can be anything from a 
living being (human, animal, etc.) to a lifeless object such as an appliance, a machine, etc. [4]. These virtual representations (VOs) of the physical world are locatable, addressable, and readable and can produce and consume services acting as a team towards a mutual goal [5]. An IoT platform consists of sub-platforms such as sensor platforms and actuator platforms. The sensor platforms are composed of sensor Vos, and the actuator platforms are composed of actuator VOs. The sensor VOs and actuator VOs are combined on the IoT platform to form various IoT services. These IoT services get the sensing data and control the IoT environments.

With the advances in the cyber-physical spaces and cyber-physical systems, many do-it-yourself (DIY) IoT platforms have been proposed in recent times [6-8]. The basic idea behind most of the DIY IoT platforms is the online availability of VOs representing physical objects in the real world. These VOs are then dragged and dropped and interconnected to produce IoT services. However, most of these offered platforms are offline, which makes them inaccessible to a large number of online users. Recently, the work presented by our co-author [9] proposed the design and implementation of a decoupled IoT application store. The application store prototype [9] allows the online discovery and sharing of VOs. Figure 1 below shows an overview of the previously proposed IoT app store [9]. The physical objects are uploaded to the application store as VOs, and each VO has an executable file that can be accessed by another online user. As the architecture below shows, multiple VOs can be joined together to form multiple microservices and then multiple IoT services. The scope of the previously published IoT app store could not cover the formation of multiple IoT services based on multiple VOs. It was limited to the uploading, discovery, and sharing of the VOs and to forming microservices associated with single VOs only.

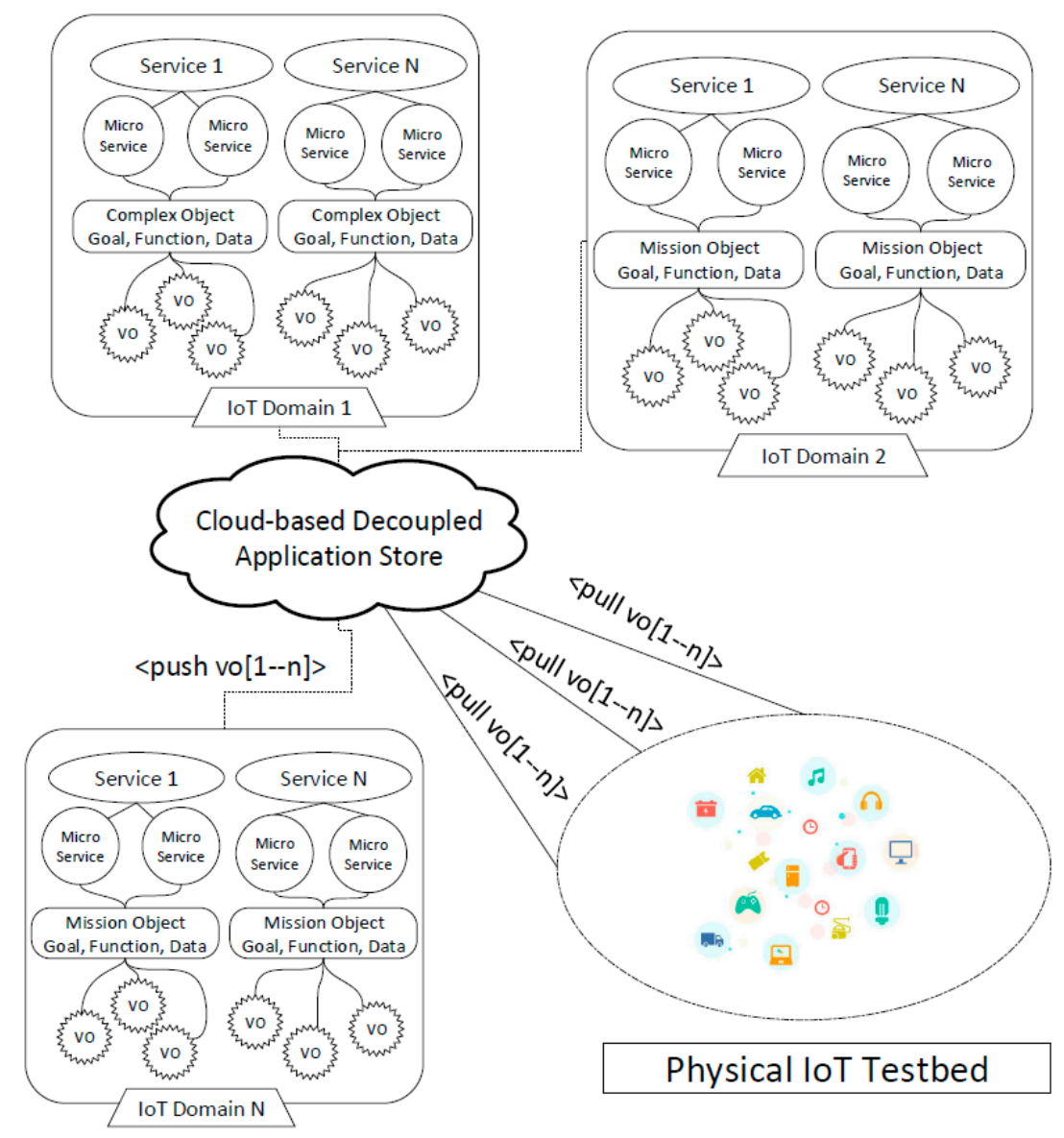

Figure 1. Overview of the architecture of the previously proposed IoT app store [9]. IoT: internet of things, VO: virtual object. 
Hence, in this paper we propose a solution to integrate the VOs on the basis of contextual information in order to produce IoT services. These IoT services can be composed of a set of Vos, each having the physical object at different physical locations.

In order to improve the readability of this article, we present the definitions of the abbreviations used in this article (Table 1).

Table 1. List of used abbreviations.

\begin{tabular}{cc}
\hline Abbreviation & Full Form \\
\hline VO & Virtual Object \\
IoT & Internet of Things \\
SN & Sensor Network \\
AN & Actuator Network \\
DIY & Do-It-Yourself \\
App Store & Application Store \\
\hline
\end{tabular}

In this work, we propose the design and implementation of fusion IoT services based on multi-physical-space VOs and IoT platforms. In the proposed solution, a sensor platform, an actuator platform, and a fusion IoT services platform are designed and developed. The sensor platform accesses all the sensors VOs from the online IoT app store, while the actuator platform accesses all the actuator VOs from the online IoT app-store. The sensor and actuator platforms perform the contextual mappings of the available sensors and actuators. The fusion IoT services platform accesses the available sensors and actuators profiles from the sensor and actuator platforms and creates the fused IoT services to be offered to the users. The contributions of the proposed work are listed below:

- Reuse of existing VOs resources

- Integration and mapping of existing VOs into meaningful scenarios

- Using the mapped scenarios to offer useful fused IoT services

- Eliminating the need of duplicate deployments

- Ease of deployment for the user to create user's own VO services based on DIY

- Reducing the total cost of the physical networks' deployment

- Reducing the total cost of the physical networks' maintenance

- Visualization of the offered services

- Extending the access of offered services to virtual users

The paper also contributes towards a sustainable solution for the service consumption of cyber-physical networks. By definition, the word sustainability refers to avoiding the depletion of existing resources; the proposed solution also aims to use the existing resources to their full potential. Hence, the proposed solution aids in minimizing duplicate deployments of the virtual networks, the deployment costs, and the maintenance costs.

The rest of the paper is organized as follows. Section 2 presents a literature review. Section 3 presents the proposed methodology for the fusion IoT services platform. Section 4 presents the implementation environment and the execution results. Section 5 presents user case scenarios. Section 6 presents performance evaluations, and Section 7 concludes the paper with a discussion.

\section{Related Works}

In this section, we present a review of the existing literature on the topic of the proposed work. The proposed work is built on a previously proposed work in which the design and implementation of an independent online IoT application store are described, presenting a novel prototype for sharing and discovery of VOs [9]. The IoT application store is based on the idea of developing applications by using IoT components. The survey presented in [4] specifies the VOs as the foundation of building blocks in IoT applications. The use and application of virtual objects are restricted to the format at the sending 
and the receiving ends of the services and applications. A generic structure for the construction of VOs is proposed by Espada et al. [10]. The authors discuss the problems in VOs due to lack of standard formats and propose a solution regardless of business logic and interaction levels of VOs.

The work presented in [11] proposes a semi-automated service composition system. The service composition is based on two parts, i.e., service goals and provider offers. The requirements of the service are named as service goals, and possible solutions to meet the requirements are the provider offers. The works presented in $[12,13]$ also offer a solution for semi-automated service compositions using semantic descriptions and ontology for web services. A cloud-based service architecture for IoT is presented by Liu et al. [14]. The authors explore the key technologies in the IoT services such as light-weighted semantic services, context-aware service discovery, and adaptive service composition models. A novel adaptive and context-aware service composition framework for IoT environments is proposed by Urbieta et al. [15]. The services are deployed and tested for IoT-based smart cities. IoT services and applications have vastly grown in the recent past. With the excess use of VOs and the development of various IoT services for diverse applications, the question of management of these IoT VOs and services arises. The work presented in [16] focuses on the management of IoT services and virtual objects in a hyper-connected things network to enable management tasks. A service-and-virtual-objects (SVOM) system prototype is implemented by the authors for the effective organization and monitoring of physical devices via virtual objects and composed IoT services.

Cloud computing is of core importance for virtual networks, virtual objects, and virtual services deployment. The design and implementation of a sensor-cloud platform for physical sensor management in cloud-of-things (CoT) environments are presented by Hang et al. [17]. The authors highlight the need of cloud computing with the increasing number of physical sensors nodes in wireless sensor networks and propose a sensor-cloud platform for addressing efficiency issues. The authors provide an efficient management solution for virtual environments and also a graphical user interface for a better user experience and remote availability. The work presented in [18] proposes the design and implementation of a cloud-centric configuration repository for DIY IoT applications. A novel implementation is presented, which enables the general users to customize the behavior of remote IoT devices by making use of the VOs. The works presented in [19-21] suggest the idea of involving the end users into the process and service building to discover and control things for a better utilization of the applications, which can be interpreted as the DIY idea. Many IoT marketplaces [22-25] works have been presented in the recent past, and many context-aware virtual objects and virtual IoT services [26-31] works have also been proposed.

\section{Current Challeneges and Motivation}

In the literature review, we noticed a major shift in the use, design, and development of virtual physical networks and virtual services. Many works have contributed towards offering virtual services to the users in order to enhance the user experience. Some works have focused on DIY, and some have focused on uploading and sharing VOs. Currently, the existing studies do not offer solutions for creating fusion IoT services by integrating the online available sensor and actuator virtual resources. On the basis of the existing studies, the users can only access VOs independently as single resources and then will have to build and develop integrated services platform themselves. Developing users' own service platforms, for each user and for each service scenario, is a hectic task. Also, services deployed via such isolated and scenario-specific platforms would be limited in functionality as well as in access.

In this work, we put our focus on integrating the concepts of DIY IoT marketplace and VOs sharing, creating fusion virtual services and virtual services access. We build a fusion IoT services platform on our already built IoT application store (app store). The IoT app store enables the sharing and discovery of IoT VOs along with microservices associated with each VO uploaded to the app store. The fusion IoT platform will enable the user to fetch the desired or all VOs from the IoT app-store and will map the available VOs to form IoT services. The user can either select all the available VOs and 
see all the possible services combinations or select the desired (DIY) services to customize the virtual services scope. To the best of the authors' knowledge, the proposed work is the first of its kind.

\section{Proposed Methodology for Fusion IoT Services Based on Multi-Physical Spaces}

In this section, we propose a methodology for fusion IoT services based on multi-physical spaces. In Section 3.1, we present the proposed fusion IoT services overall design and architecture. Section 3.2 presents the online IoT app store's basic functionality description. Section 3.3 presents the sensor and actuator platforms' role in the proposed architecture. Section 3.4 presents the fusion IoT services platform configurations, and contextual scenario mappings are elaborated in Section 3.4.1.

\subsection{Fusion IoT Services Design and Architecture}

In this subsection, we present the conceptual diagram of the proposed idea; we consider multiple physical IoT spaces consisting of multiple sensor and actuator networks (Figure 2). The virtual IoT services are formed by combining the existing network's resources from multiple physical spaces. The idea is to amalgamate the virtual network services on the basis of the user requirements, by reusing the existing resources into users' contexts and scenarios. We aimed to explore the unlimited potential scenarios that can be discovered by mapping the sensors' and actuators' profiles from different physical zones, without having to physically deploy sensors and actuators for each scenario. The proposed idea targets the reuse of existing resources and aims at eliminating redundancy, which can reduce the total cost of the physical networks' deployment and maintenance.



Figure 2. Conceptual diagram for extracting fusion virtual IoT services from multiple physical IoT spaces.

Figure 3 presents the fusion virtual IoT services platform architecture using virtual objects based on online app store and sensor-actuator platforms. The proposed system consists of three layers, i.e., an application layer, a service layer, and a physical IoT layer. The application layer comprises the service admin and the users who access the services from the IoT app store. The IoT app store has many sensor and actuator virtual objects available, ready to be accessed by the users. The admin 
manages the virtual objects and the offered services in the IoT app store. The service layer provides the implementation of the IoT service platform, sensor platform, and actuator platform. The profile data from the sensor platform and the actuator profile data from the actuator platform are passed onto the IoT service platform for creating virtual objects and virtual services. The sensor platform creates the sensor profile data, and the actuator platform creates the actuator profile data based on the data received from the sensor and the actuator networks, respectively. The physical IoT layer consists of the sensor networks and the actuator networks, which may comprise multiple sensor and actuator nodes, varying depending on the size and number of IoT networks.

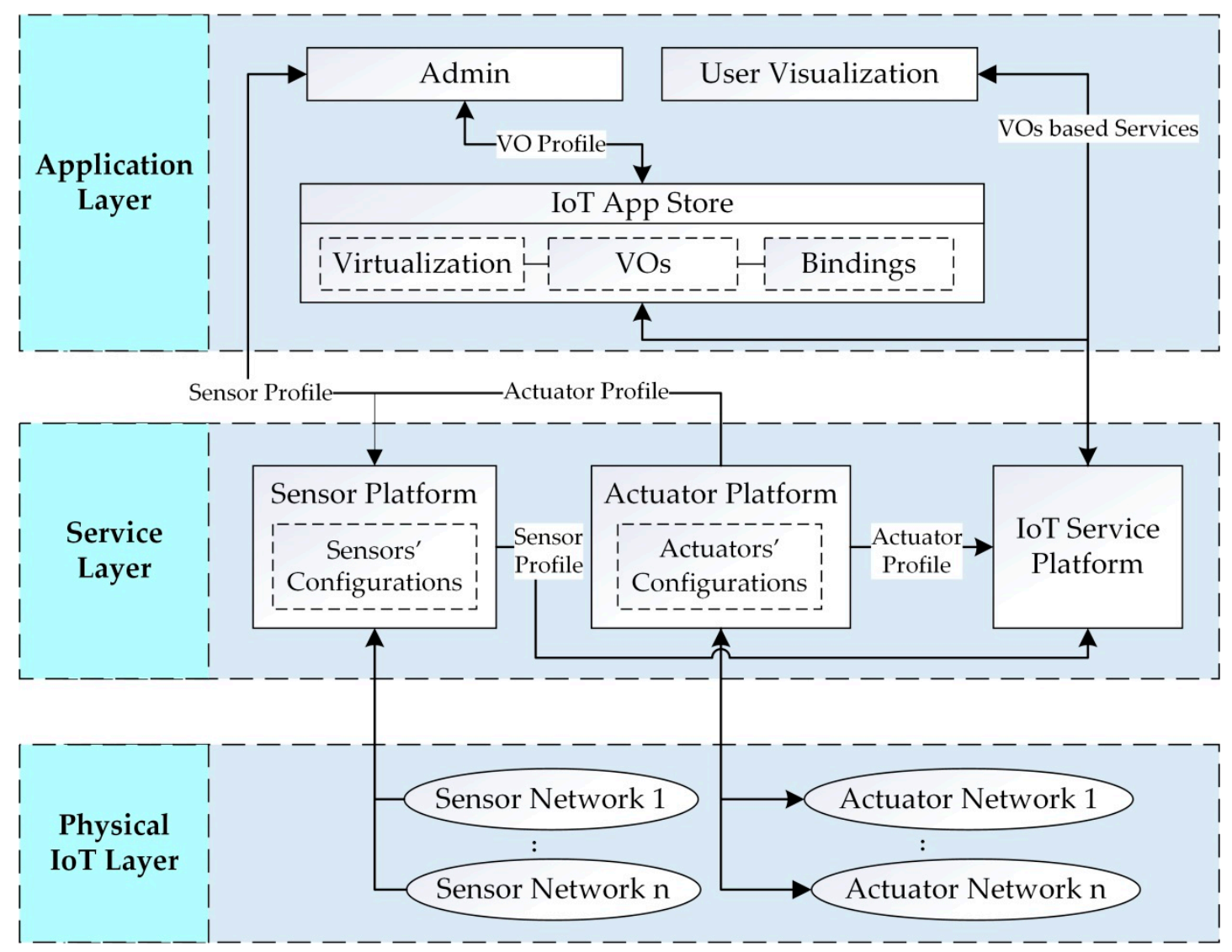

Figure 3. Advanced IoT service architecture using virtual objects based on an online app store and sensor-actuator platforms.

\subsection{Online IoT App Store}

In this subsection, we present the basic description of an online IoT app store. An online IoT app store is a platform where multiple users upload the virtual representations (VOs) of physical IoT objects (sensors objects and actuators objects). In this work, we used a previously established online IoT store [9]. The VOs were uploaded along with their name, IP (Internet Protocol) address, description, and executable file, which enables the users to access the actual object via the given VO, that is, access the data from the physically installed sensor or control the physically installed actuator. The user accessing the VO can be at a location $X$, and the physical sensor or actuator object can be at another location Y (Figure 4). 


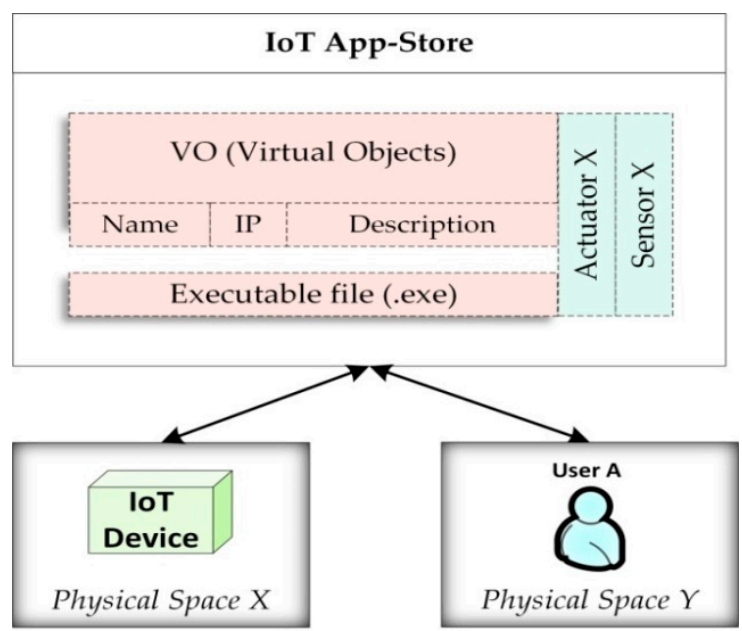

Figure 4. Virtual objects upload and access from the IoT app store. IP: Internet Protocol address

\subsection{Sensor and Actuator Platforms for IoT App store}

In this subsection, we present the role of sensor platform and actuator platform in the proposed architecture for fusion IoT services.

The sensor platform fetches the sensor VOs from the online IoT app store and parses the available information to form a sensor profile for each accessed sensor object. Similarly, the actuator platform creates the actuator profile for each of the fetched actuator VOs. The sensor platform and the actuator platform also have additional options to configure any additional sensors/actuators from the users' personal sensor/actuator networks, which might not be available at the online IoT app store or the user might want not to make those objects public by uploading them in the app store. The sensors and actuators are connected to the platform via an on-platform middleware module which performs sensor/actuator configurations and IP/ID mappings for further creating the sensor/actuator profile. Where, IP refers to device's IP address and ID is the unique identifier of each device (Figure 5).

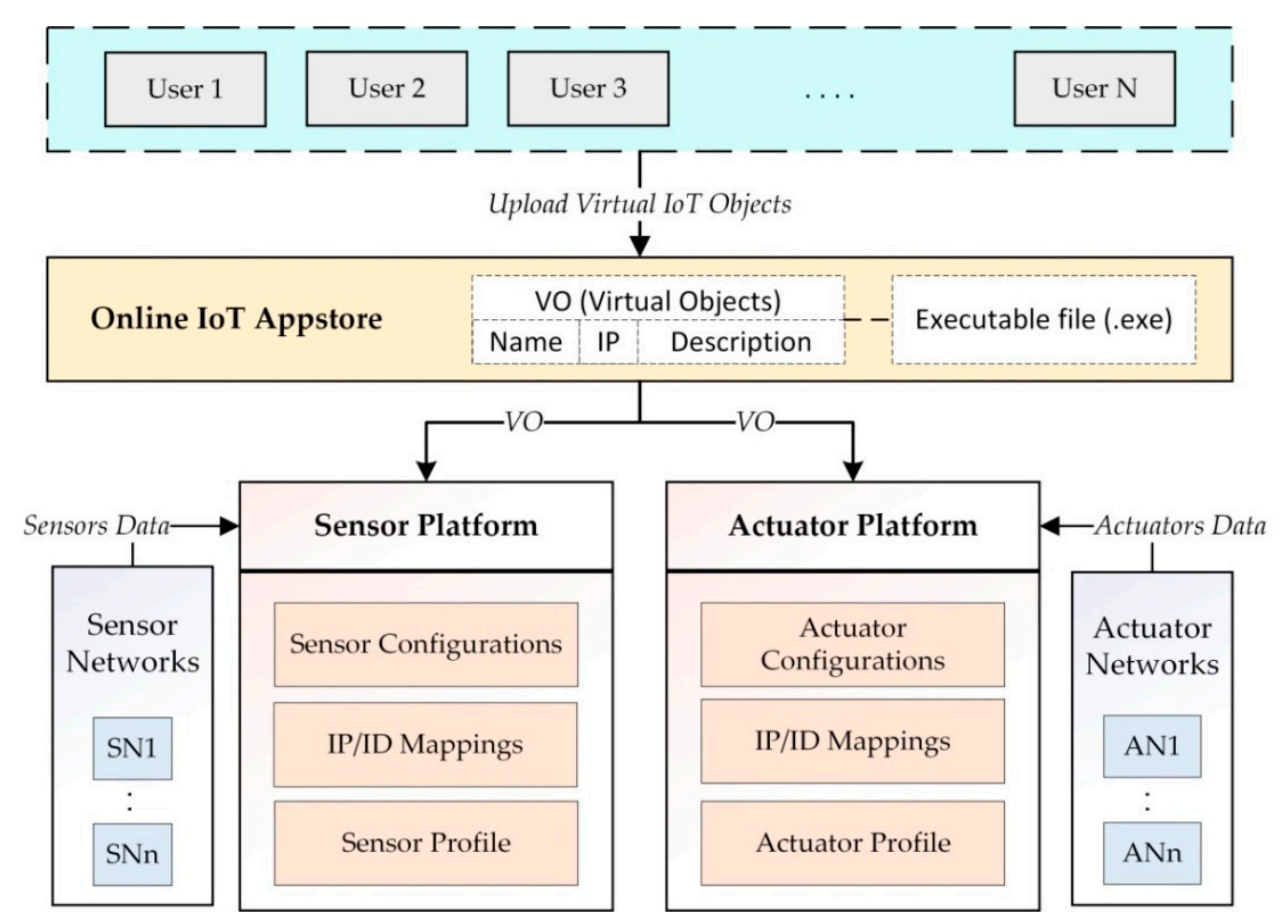

Figure 5. Sensor and actuator platforms for the IoT app store. SN: sensor network, AN: actuator network, IP/ID: internet protocol address/identifier 
The sensor and actuator profiles contain details such as the device ID, device type, device name, data type, actions available to be performed at the device status, and controls available for the device.

\subsection{Fusion IoT Services Platform}

In this subsection, we present the design and details of the fusion IoT services platform. In Figure 6, the configurations of the fusion IoT services platform are presented. The IoT services platform receives the sensor profiles and actuator profiles from the sensor platform and actuator platform, respectively. Once the sensors' and actuators' features are extracted from the sensor and actuator profiles, the IoT services platform performs the sensor readings mapping into use cases for the available sensors data, as well as the control function mapping into use cases for the available actuators data. Next, it performs and analyzes the hybrid sensors and actuators contextual mapping to form IoT scenarios based on different combinations of multiple sensors and actuators. The resulted IoT scenarios are then composed into fusion IoT services and deployed in the IoT services platform's visualization unit. The deployed services have different access rights based on the origin and permissions given by the publisher of the VOs involved in the fusion IoT services.

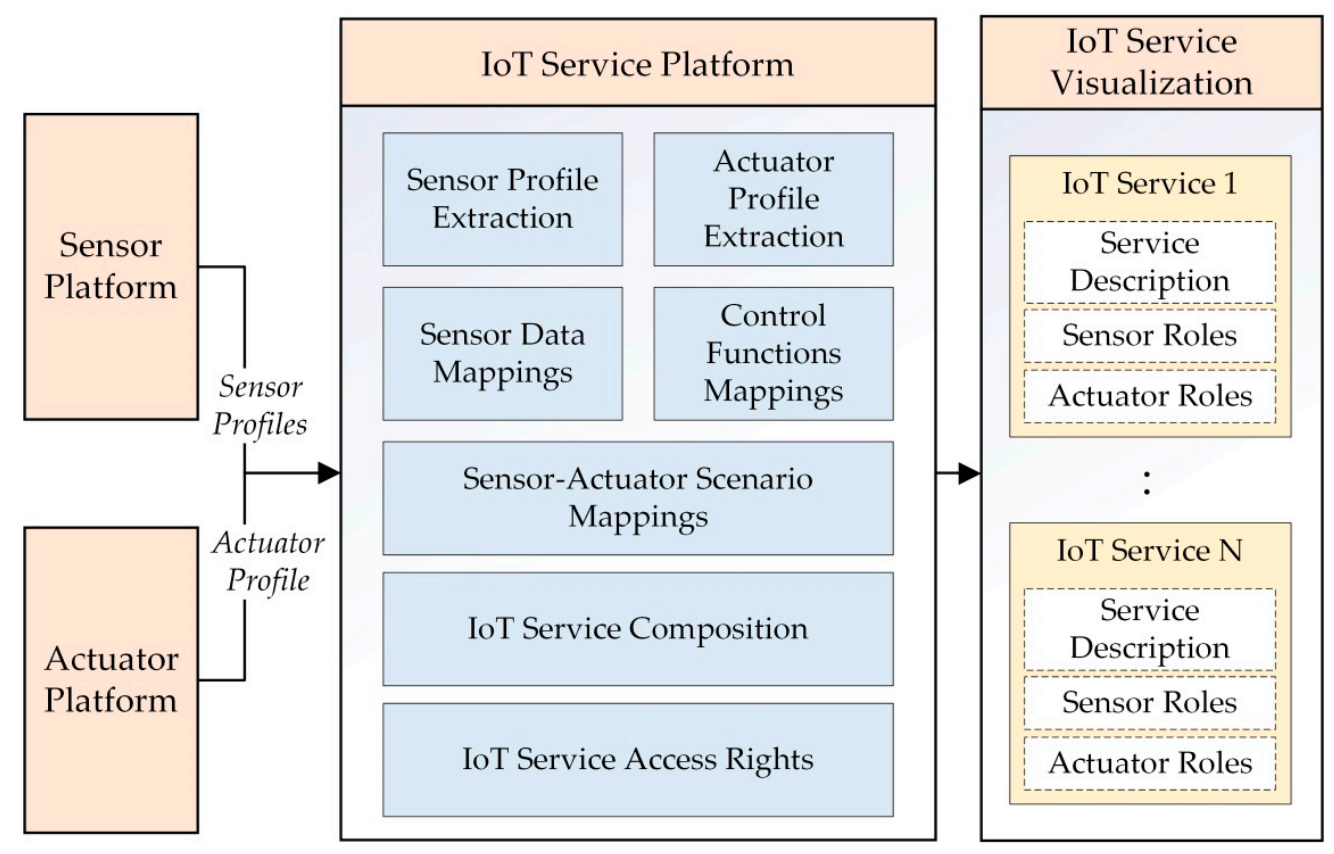

Figure 6. Configurations diagram for the fusion IoT service platform and service visualization.

\subsubsection{Contextual Scenario Mappings}

In this section, we describe the contextual scenario mappings of sensor and actuator VOs. Multiple sensor VOs and actuator VOs are available for the fusion IoT services platform via the IoT app store. The fusion IoT services platform extracts the contextual information from the sensors' profile data and the actuators' profile data, and then this contextual information is used to make scenarios based on multiple sensor and actuator VOs (Figure 7). 


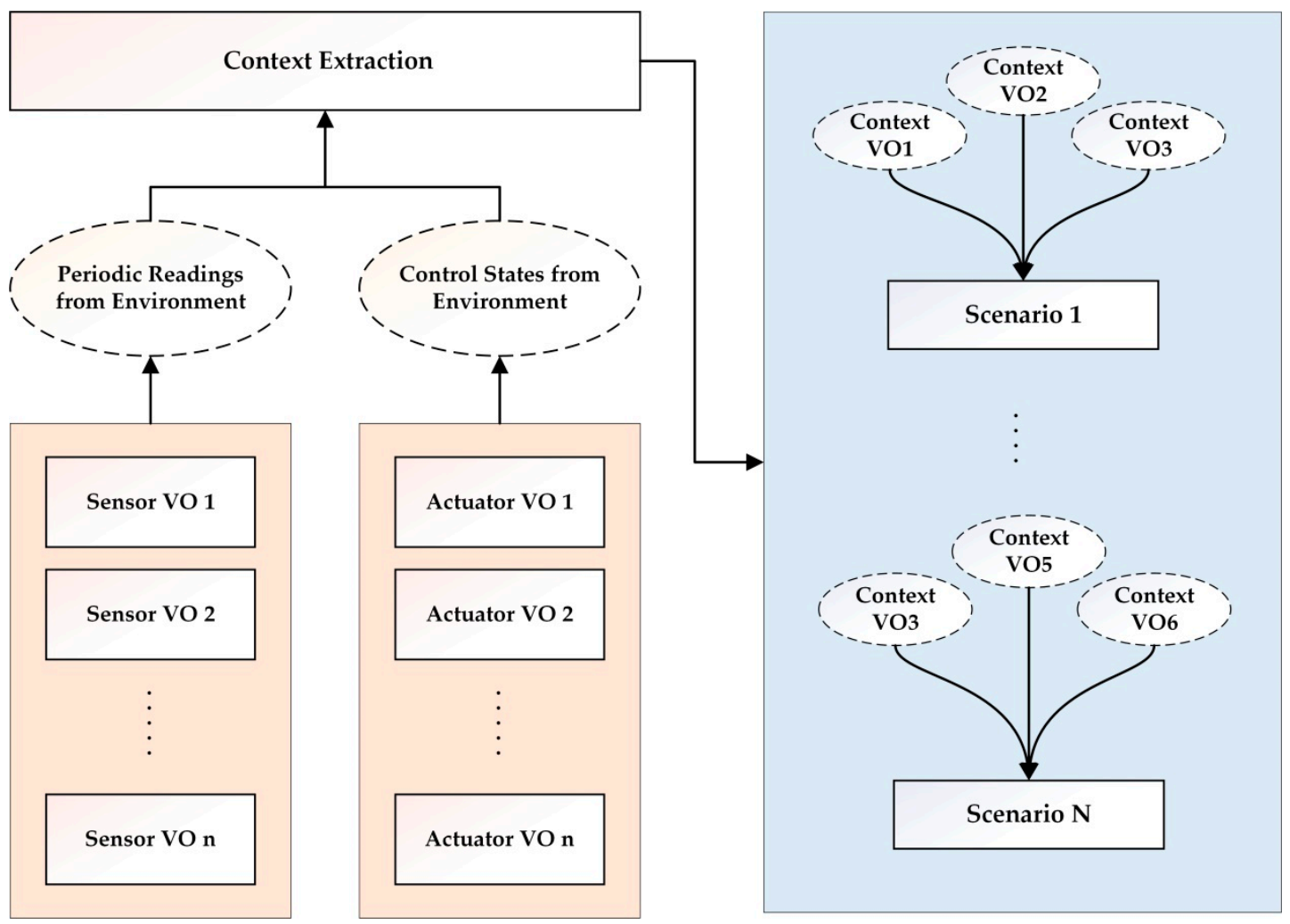

Figure 7. Contextual extraction and scenario formation from VOs.

The contextual information is extracted on the basis of attributes such as VO type, current environment type of the $\mathrm{VO}, \mathrm{VO}^{\prime}$ s value type, $\mathrm{VO}^{\prime} \mathrm{s}$ value ranges, reading interval in the case of sensors' $\mathrm{VO}$, access rights to the $\mathrm{VO}$, dependencies associated and relations associated with the VO. Once the contextual information is extracted, multiple VOs are then combined on the basis of their common attributes and resolved dependencies and relations. Multiple VOs are combined and mapped into forming scenarios. Multiple scenarios are then mapped into multiple environments to form fusion IoT services based on the scenario context and environmental context (Figure 8).

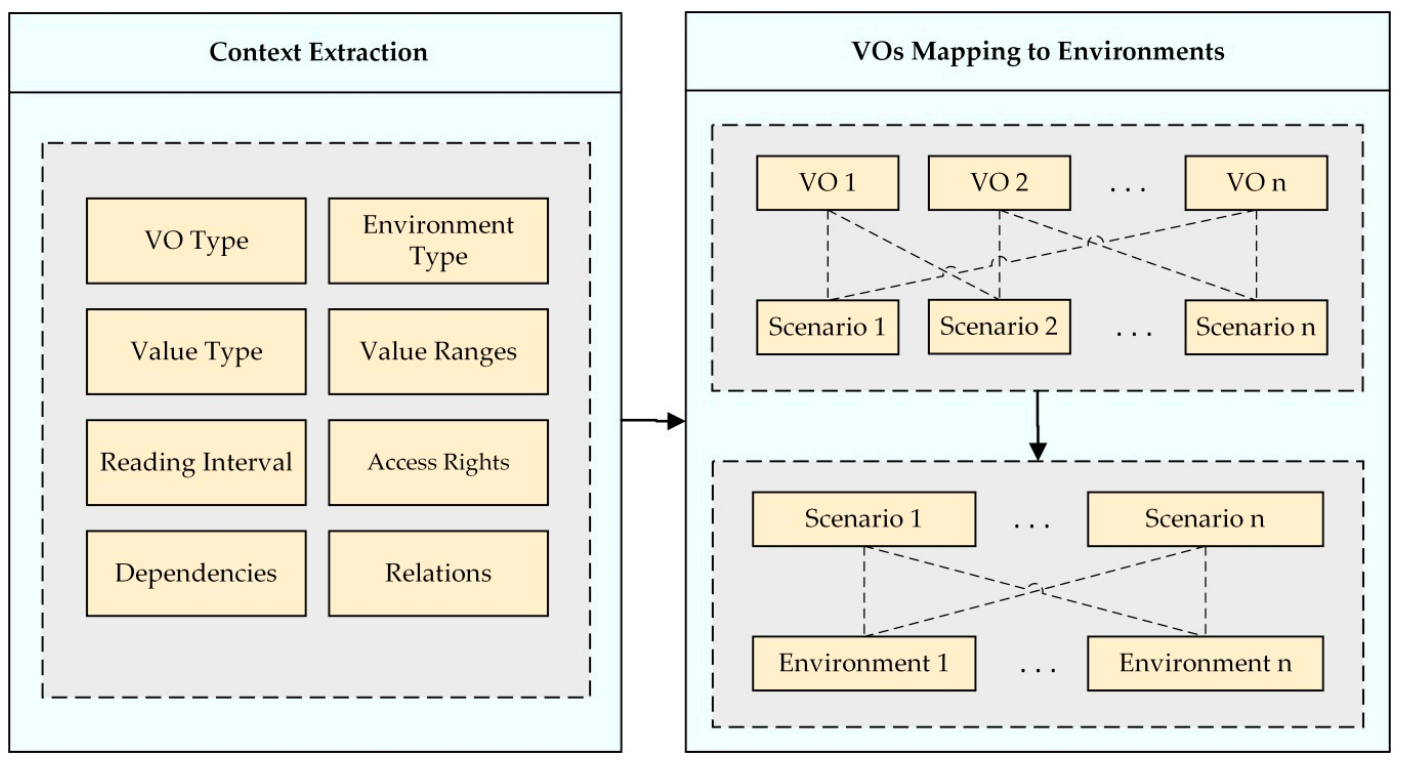

Figure 8. VOs mapping into scenarios and environments on the basis of contextual information. 


\section{Implementation and Execution of the Proposed Fusion IoT Services Platform}

In this section, we present the implementation and execution of the proposed system. In Section 4.1, we discuss the implementation setup. In the Section 4.2, we present the overview of our implemented service execution results and visualization.

\subsection{Implementation Setup}

In this section, we present the implementation environment of the proposed work. Table 2 below shows the detailed implementation environment. The operating systems used are windows and Linux, and the primary languages used are C sharp and PHP. PHP (Hypertext Preprocessor) is a popular general-purpose scripting language. The fusion IoT services platform was developed in visual studio .NET framework developed by Microsoft, while the IoT app store was developed using Drupal 8, which is an open-source framework. The database used is MySQL.

Table 2. Implementation environment for the fusion IoT services platform.

\begin{tabular}{ll}
\hline \multicolumn{1}{c}{ System Component } & \multicolumn{1}{c}{ Value } \\
\hline Operating System & Windows; Linux \\
\hline CPU & Intel @Core ${ }^{\mathrm{TM}}$ i5-4570 CPU at 3.20 GHz \\
\hline Primary Memory & $8 \mathrm{~GB}$ \\
\hline Programming Language & C \# and PHP \\
\hline Framework & .NET framework and Drupal 8 \\
\hline Libraries & HTTP foundation, ReactJS, HTTPKernal, Bootstrap 3, \\
\hline Database & HTML 5/CSS3, TWIG, Apache Jena \\
\hline
\end{tabular}

\subsection{Execution Results and Visualization}

Figure 9 shows the execution screen of the IoT app store. The app store has multiple VOs uploaded, representing the sensor and actuator objects. Each VO has its description such as title, model, type, methods available, supported protocols, and an executable file which can be used to access the VO. The execution screen in the given image shows the uploaded VOs for the actuator objects.

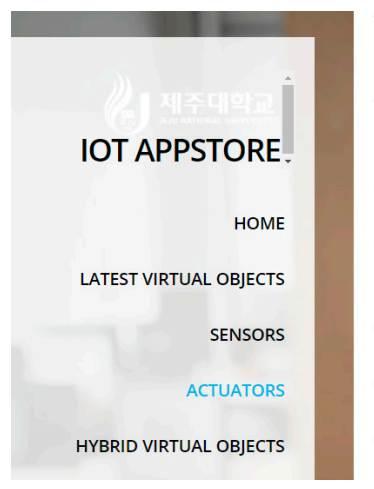

\begin{tabular}{|c|c|c|c|c|c|c|}
\hline THUMBNAIL & TITLE & MODEL & $\begin{array}{l}\text { vo } \\
\text { TYPE }\end{array}$ & ATTRIBUTES & METHODS & $\begin{array}{l}\text { SUPPORTED } \\
\text { PROTOCOLS }\end{array}$ \\
\hline & $\begin{array}{l}\text { Alexa Echo Dot - } \\
\text { Sehrish }\end{array}$ & SmartHome & Actuator & & On, Off & HTTP \\
\hline & Light-Talha & light-talha & Actuator & & light_on, light_off & COAP \\
\hline & LED from Asif (new) & led-asif-new & Actuator & & $\begin{array}{l}\text { asifs_LED_On, } \\
\text { asifs_LED_Off }\end{array}$ & COAP \\
\hline & LED from Faisal & led-fsl & Actuator & & & \\
\hline & LED from $\mathrm{hl}$ & RedLED & Actuator & & On & COAP \\
\hline & LED from $\mathrm{hl}$ & RedLED & Actuator & & On & COAP \\
\hline
\end{tabular}

Figure 9. IoT app store for VO deployment.

Figure 10 shows the execution screens for sensor and actuator platforms. In Figure 10a, a connection with the IoT app store is established. In Figure 10b, the sensor platform is shown, which performs the sensor configurations and ID/IP mapping for new sensors to be added; the available sensor VOs from the IoT app store are also shown. Similarly, in Figure 10c, the actuator platform is shown, which performs the actuator configurations and ID/IP mapping for new actuators to be added; the available actuators VOs from the IoT app store are also shown. Sensor platform and 
actuator platform create the sensor profiles and the actuator profiles to be forwarded to the fusion IoT services platform.

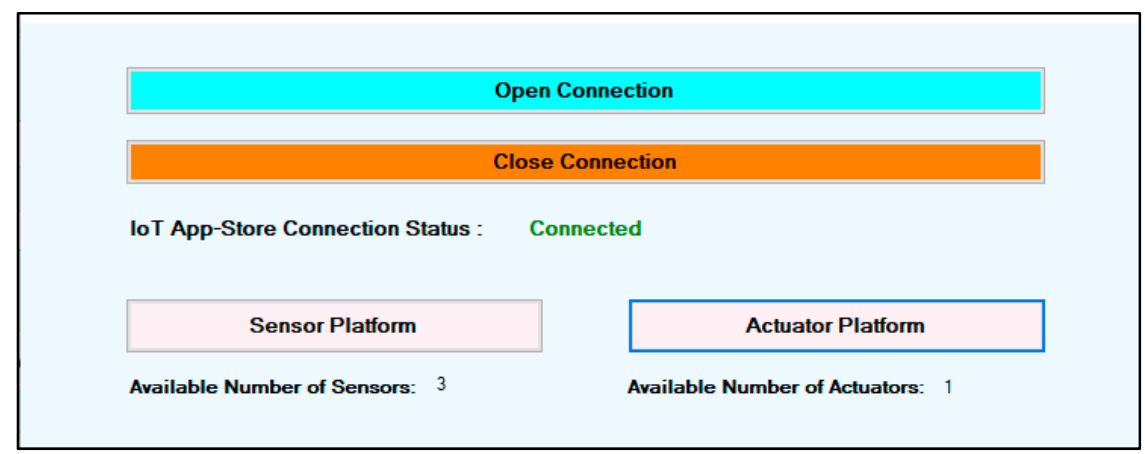

(a) connection establishment with the IoT app store

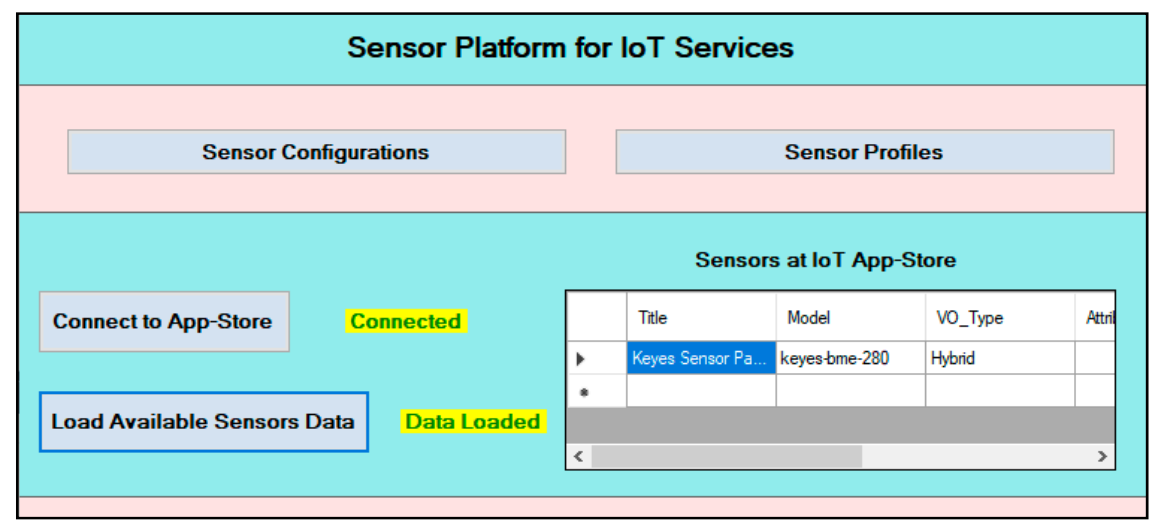

(b) with the sensor platform for IoT services

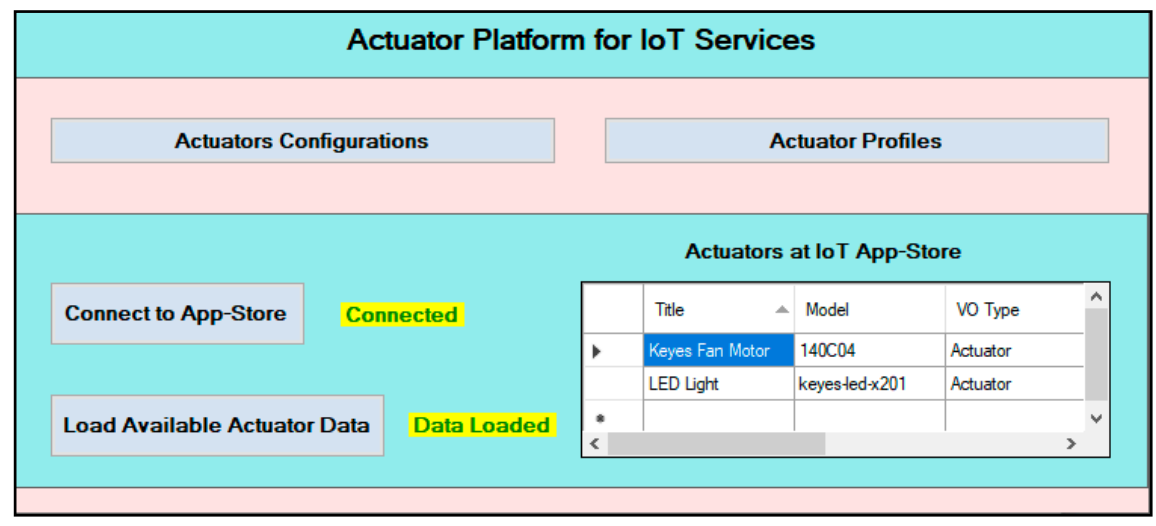

(c) with the actuator platform for IoT services

Figure 10. Establishment and features of the connection of the sensor platform and actuator platform.

Figure 11 shows the fusion IoT services platform, where sensor profiles and actuator profiles are mapped into different contexts to provide multiple services to the users based on the user's requirements. The services are provided by accessing the available VOs and finding useful contexts from them. The system offers services such as multi-location route predictions, plant growth-rate predictions, and smart space surveillance. The services are created on the basis of the contextual mappings of the sensors and the actuators in order to derive meaningful scenarios. The services can be accessed by the users and can be useful in many different applications and research studies. 


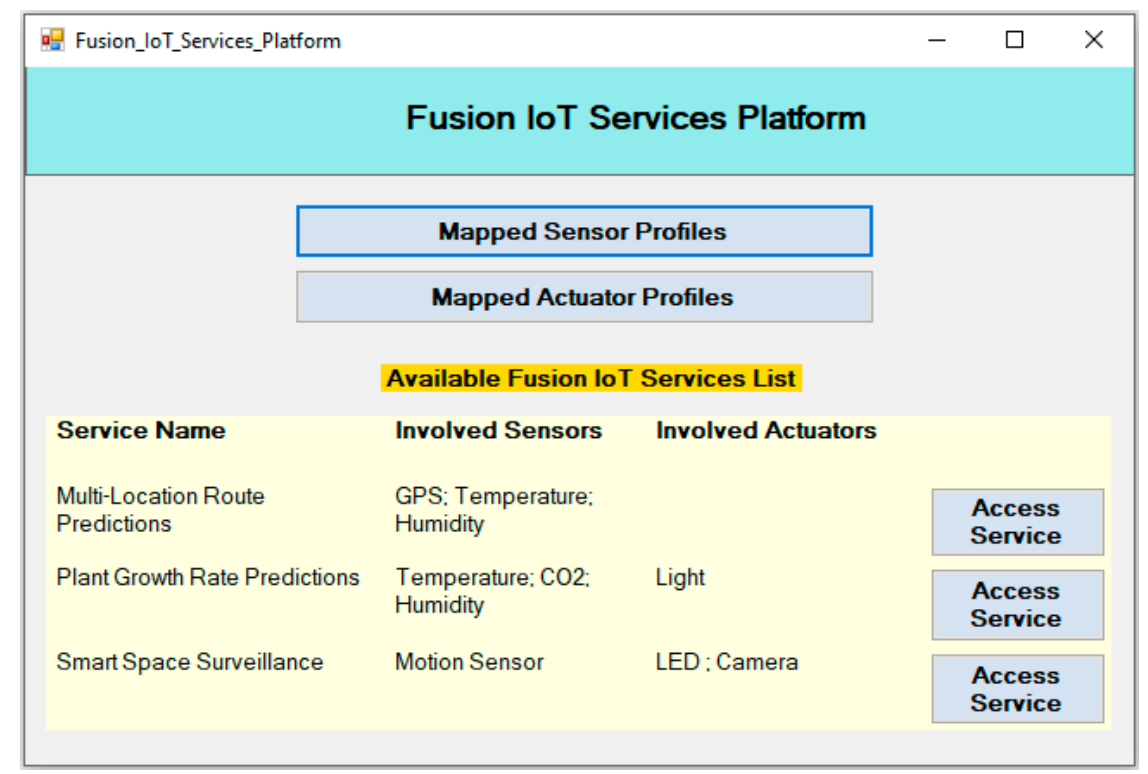

Figure 11. Fusion IoT services platform execution screen for service description and access.

Accessing the service will provide the user with periodic sensing data streaming from the VOs involved and the control of actuator VOs based on the access rights by the VOs publisher.

\section{Use Case Scenarios}

In this section, we present use case scenarios for better understanding the proposed system.

\subsection{Multi-Location Route Prediction}

In this sub-section, we present a use case scenario of multi-location route prediction using the proposed system.

We will consider a scenario where multiple smart cars' users have created and uploaded the VOs of the sensors installed in their smart cars (Figure 12). The sensing data received can be weather readings, location readings, traffic readings, or car health readings. The fusion IoT services platform maps the sensors' profiles and involved actuators' profiles on the basis of the extracted locations and creates single route prediction services and overlapping route prediction services for multiple locations (e.g., different cities and zones) and various conditions. Such service can be used by other travelers, prediction application developers, and researchers (Table 3).

Table 3. Route prediction service's potential consumers and description.

\begin{tabular}{ll}
\hline \multicolumn{1}{c}{ Service Consumer } & \multicolumn{1}{c}{ Service Description } \\
\hline Travelers & $\begin{array}{l}\text { Service directly accessed by a traveler to know the } \\
\text { current traffic status, weather conditions for driving, } \\
\text { or popular visited sites in a specific city or zone. }\end{array}$ \\
\hline Prediction Application Developers & $\begin{array}{l}\text { The service can be very useful for multiple route } \\
\text { prediction or smart car-based prediction applications. }\end{array}$ \\
\hline Researchers & $\begin{array}{l}\text { The data gathered and recorded by such VOs and } \\
\text { services combinations can be of huge importance for } \\
\text { researchers interested in the field of constraint-based } \\
\text { route predictions. }\end{array}$ \\
\hline
\end{tabular}




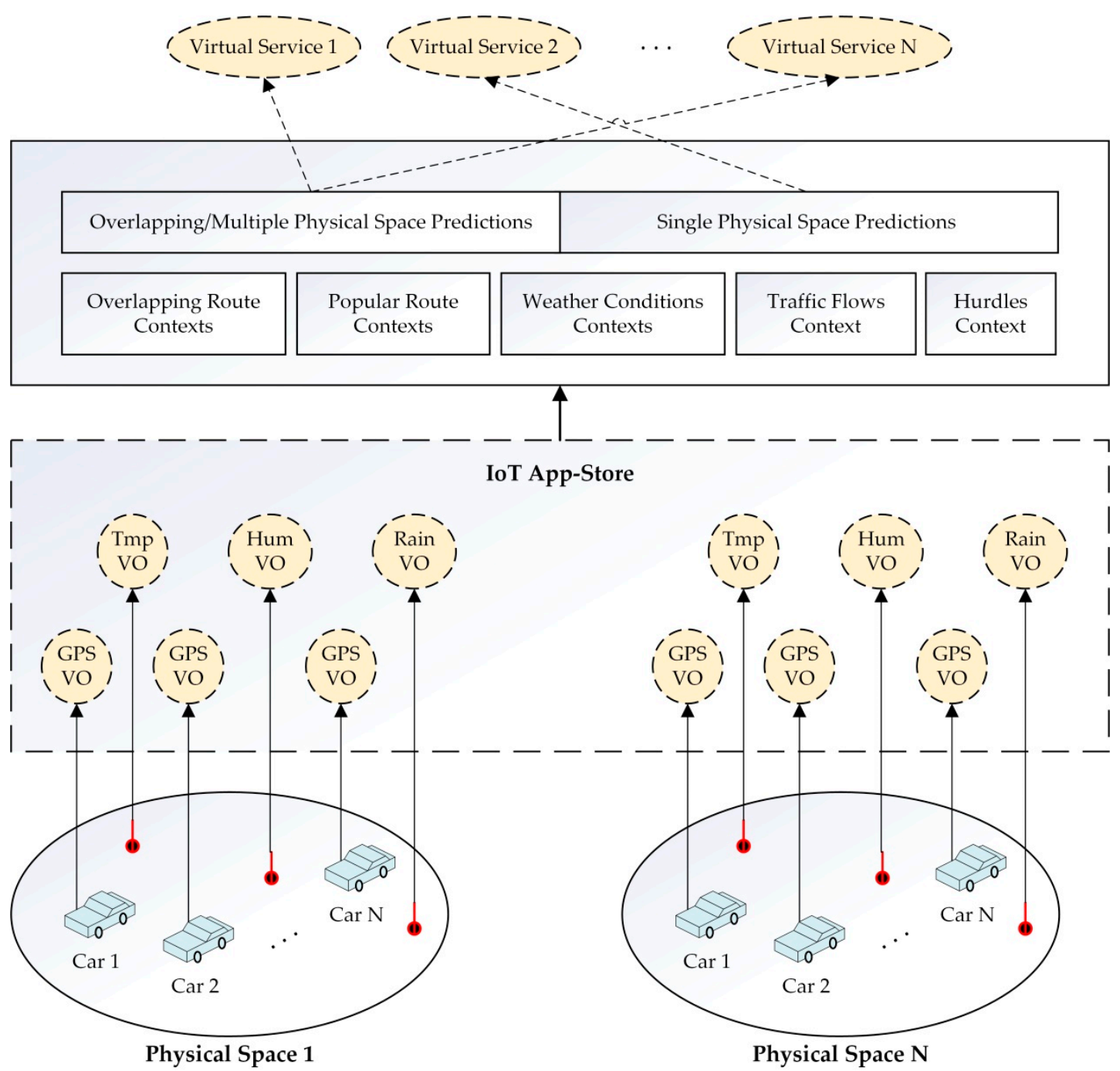

Figure 12. Use case scenario for route prediction fusion IoT services.

\subsection{Plant Growth-Rate Predictions}

In this sub-section, we present a use case scenario of plant growth rate in greenhouses. The scenario proposes two solutions, i.e., plant growth-rate prediction and optimal growth-rate parameters settings (Figure 13).

The combination of IoT and greenhouses is acquiring high importance with the emergence of new technologies. Many greenhouses across the globe are integrating IoT to improve and optimize the greenhouse environment. We consider a scenario where multiple smart greenhouses' sensors and actuators are uploaded as VOs onto the IoT app store. The proposed system will map and extract the plant growth-rate services for prediction and optimal parameter settings based on plant type and environmental parameter settings such as temperature, humidity, and CO2. The beneficiaries of such services include greenhouse businesses and researchers in the field (Table 4). 
Table 4. Greenhouse service's potential consumers and description.

\begin{tabular}{ll}
\hline \multicolumn{1}{c}{ Service Consumer } & \multicolumn{1}{c}{ Service Description } \\
\hline Greenhouse Businesses & $\begin{array}{l}\text { Independent greenhouse businesses can access and view } \\
\text { the optimal parameters for plant growth based on the } \\
\text { specifications such as plant type, environmental } \\
\text { parameters, geographical constraints, etc. }\end{array}$ \\
\hline Researchers & $\begin{array}{l}\text { The greenhouse data gathered and recorded by such VOs } \\
\text { and services combinations can be of huge importance for } \\
\text { researchers interested in the field of prediction, } \\
\text { optimization, and control of greenhouses. }\end{array}$ \\
\hline
\end{tabular}

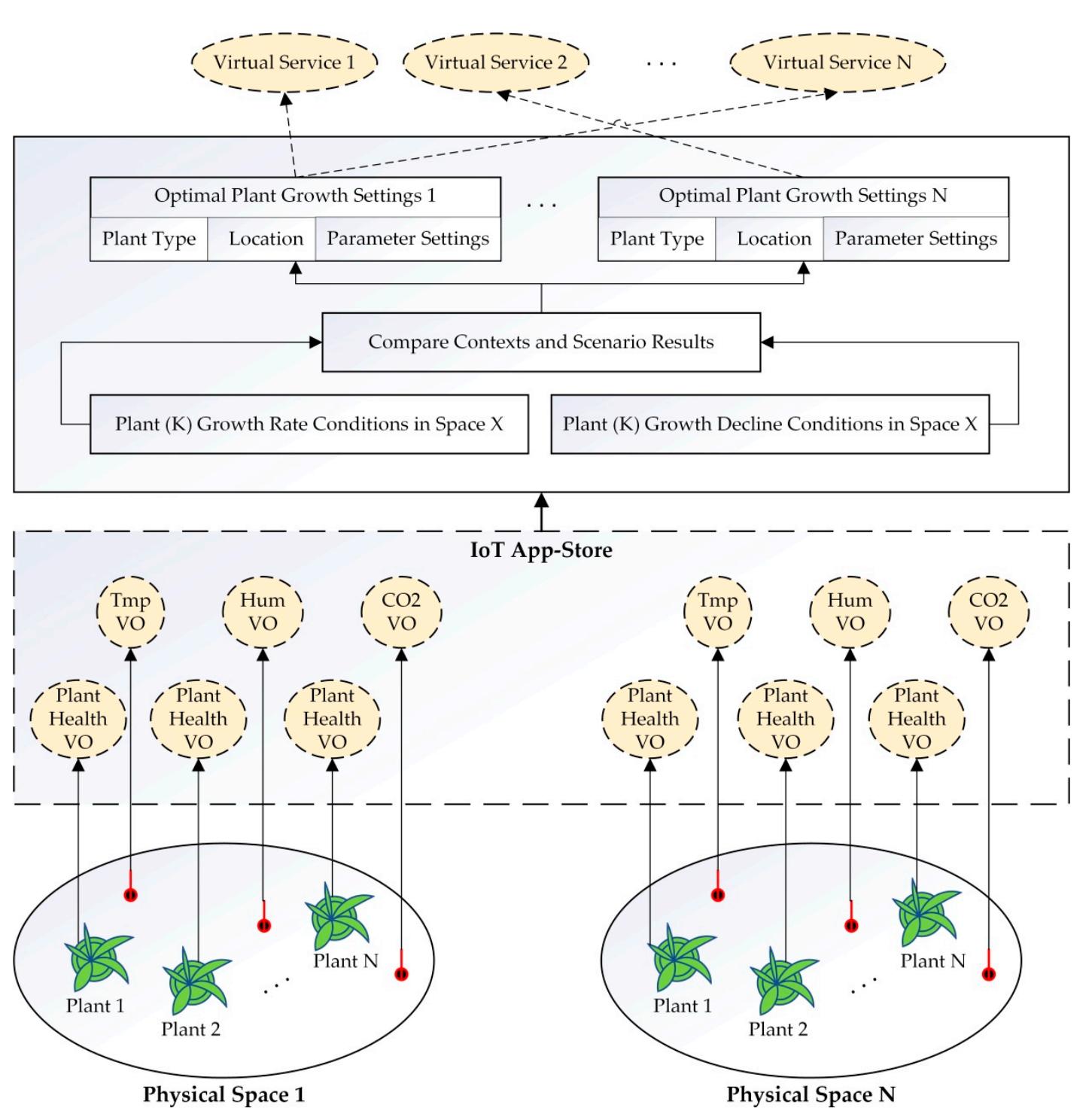

Figure 13. Use case scenario for greenhouse plant growth fusion IoT services.

\section{Performance Evaluation}

In this section, we present the performance evaluation of the proposed methodology for fusion IoT services based on multi-physical spaces. We evaluate the proposed system on the basis of the performance parameters, service connection times, service response times with varying load of VOs, virtual users, and active platforms. 
In Figure 14, we present the simulation results of fusion IoT services' connection times based on the number of virtual users. Multiple virtual users make attempts to access multiple IoT services from the fusion IoT services platform. The service connection times are shown on the primary y-axis, and the varying service instances on the secondary y-axis. We can observe that based on the service instances load accessed by a user, there's a gradual but increase in the service connection time. The connection time for requests of multiple services' access by multiple users takes about $574 \mathrm{~ms}$ on average. Depending on the varying load-multiple access requests by multiple users-an average of $300 \mathrm{~ms}$ is clearly an acceptable connection time for the system.

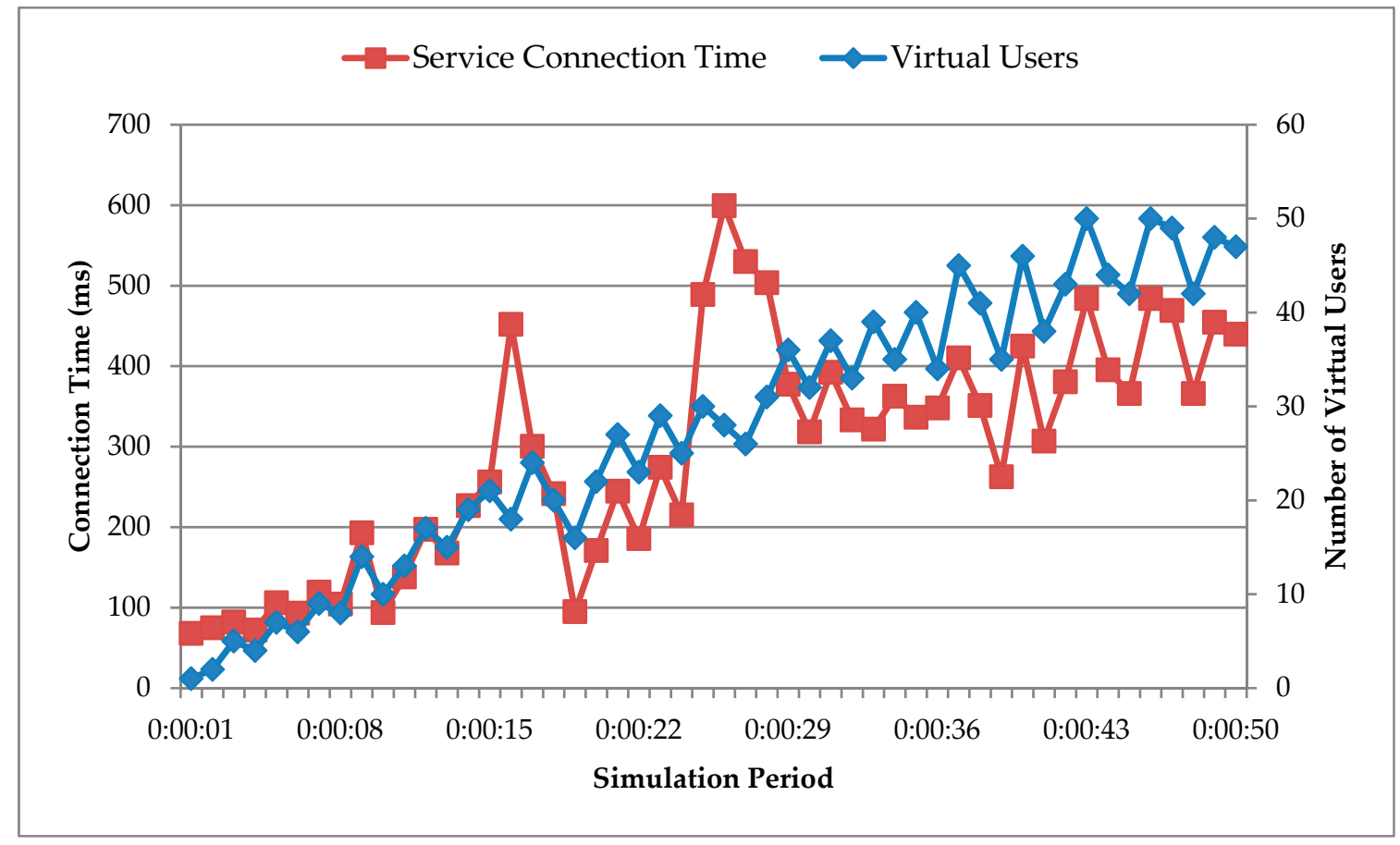

Figure 14. Fusion IoT services' connection time based on the number of accessed service instances.

In Figure 15, we evaluate the fusion IoT services' response time based on the number of VOs involved in the service and the number of active fusion IoT service platforms. We performed the simulations with 1-12 fusion IoT service platforms and 1-50 VOs. Each fusion IoT platform fetched the VOs from the IoT app store, created the sensor VO profiles and actuator VO profiles, and then produced the list of services based on the contextual mappings. The response time to service offerings varied on the basis of the number of VOs, VOs contexts, and active platforms. We obtained an average response time of $763.93 \mathrm{~ms}$ based on our simulations. 


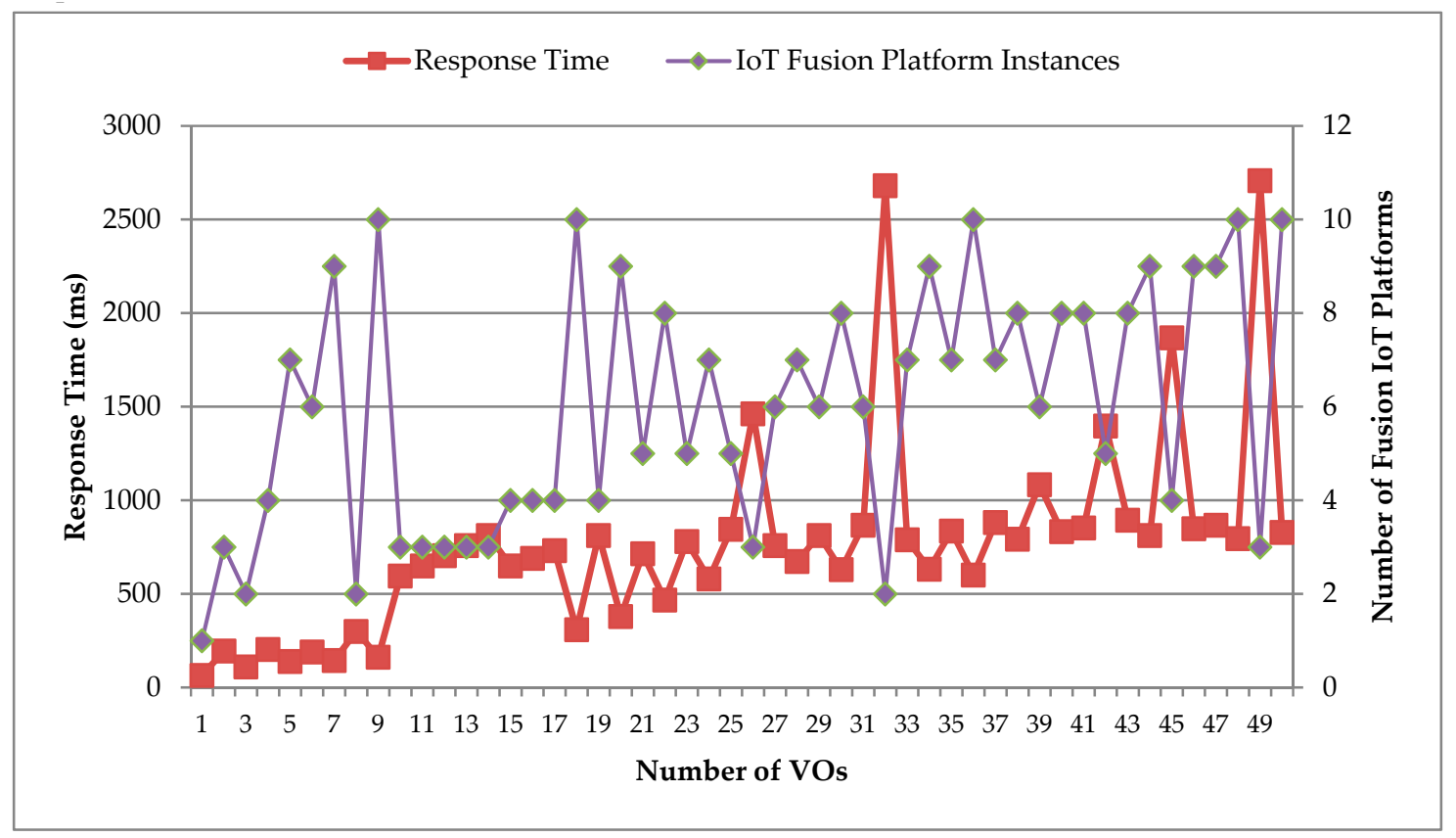

Figure 15. Fusion IoT services response time depending on the number of VOs and fusion IoT platform instances.

\section{Discussions}

Recently, the concepts of IoT marketplace, DIY IoT platforms, VOs, and virtual services have been hot topics of research in IoT virtual world. In this work, we studied in depth the aforementioned concepts and their recently proposed solution for virtual services consumption. In our literature review, we noticed the lack of sustainable solutions for fulfilling the users' multiple service consumption requirements. The proposed work focused on maximizing resource utilization, minimizing the need of duplicate virtual networks' deployments, and minimizing deployment and maintenance costs. Hence, we propose the integration of these concepts to offer fusion IoT services to the virtual users. In this paper, we propose a novel approach to a fusion IoT services platform based on virtual objects from an online application store using sensor and actuator platforms in multi-physical IoT spaces. In this work, we aimed to make use of physical objects residing in multiple spaces deployed as VOs at the IoT app store in order to create fusion IoT services. The fusion IoT services reuse the VOs into multiple contexts and multiple scenarios. The detailed design of the fusion IoT services platform is presented, and the platform was implemented to demonstrate use case scenarios based on the sensor and actuator VOs.

The performance of the system was evaluated on the basis of technical merits such as varying VOs load, varying fusion IoT platforms load, and varying the virtual users' number. The load of VOs is defined by the number of existing VOs available at the online IoT app store, which are being fetched and mapped at the fusion IoT services platform. The fusion IoT platforms load refers to the number of active platforms which are fetching the VOs data from the online IoT app store and are in the process of offering the fusion IoT services. The virtual users load refers to the number of users which are accessing the IoT app store and fusion IoT services platform for service consumption. The performance metrics used for results analyses were service connection times and service response times. The results achieved in the simulations proved that the system performance is acceptable even in the highest load conditions.

The proposed fusion IoT services platform can be used by the end-users depending on their requirements. The created services and gathered data can be used by prediction applications, optimization applications, and control applications based on specific scenarios. The composed fusion services and gathered data can also be used by researchers for simulation purposes, comparisons, and analysis purposes and survey purposes. 
The limitations of the proposed system include the dependency of the IoT service's scenario building on VOs availability in the online app store. If a user specific scenario's sensor/actuator virtual objects are not available in the online app store, then the IoT service for the required scenario cannot be composed. In such cases, the user will have to either self-deploy and upload the required VOs or wait for some other users to upload the required VOs. To overcome such limitations, in the future we plan to research more in the proposed direction and extend our project, so to propose possible solutions for such circumstances.

Author Contributions: Data curation, S.M.; Formal analysis, S.M.; Funding acquisition, D.K.; Investigation, S.M., S.A.; Methodology, S.M.; Resources, D.K.; Software, S.M.; Supervision, D.K.; Validation, S.M.; Visualization, S.M., S.A.; Writing—original draft, S.M.; Writing—review \& editing, D.K.

Acknowledgments: This research was supported by Energy Cloud R\&D Program through the National Research Foundation of Korea (NRF) funded by the Ministry of Science, ICT (2019M3F2A1073387), and this research was supported by Institute for Information \& communications Technology Planning \& Evaluation(IITP) grant funded by the Korea government(MSIT) (No.2018-0-01456, AutoMaTa: Autonomous Management framework based on artificial intelligent Technology for adaptive and disposable IoT). Any correspondence related to this paper should be addressed to Dohyeun Kim.

Conflicts of Interest: The authors declare no conflict of interest.

\section{References}

1. Atzori, L.; Iera, A.; Morabito, G. The internet of things: A survey. Comput. Netw. 2010, 54, 2787-2805. [CrossRef]

2. Buckley, J. Conference Report: From RFID to the Internet of Things; Pervasive Networked Systems CCAB: Brussels, Belgium, 2006.

3. Sundmaeker, H.; Guillemin, P.; Friess, P.; Woelfflé, S. Vision and Challenges for Realising the Internet of Things; Cluster of European Research Projects on the Internet of Things; European Commision: Brussels, Belgium, 2010; Volume 3, pp. 34-36.

4. Nitti, M.; Pilloni, V.; Colistra, G.; Atzori, L. The virtual object as a major element of the internet of things: A survey. IEEE Commun. Surv. Tutor. 2016, 18, 1228-1240. [CrossRef]

5. Roman, R.; Najera, P.; Lopez, J. Securing the internet of things. Computer 2011, 9, 51-58. [CrossRef]

6. Cloutier, G.; Papin, M.; Bizier, C. Do-it-yourself (DIY) adaptation: Civic initiatives as drivers to address climate change at the urban scale. Cities 2018, 74, 284-291. [CrossRef]

7. Valderrama, C.; Vachaudez, J.; Bettens, F.; Vinci dos Santos, F.; Menezes, N.; Roelands, M. The DiY Smart Experiences Project: A European Endeavour Removing Barriers for User-generated Internet of Things Applications. In Architecting the Internet of Things; Springer: Berlin/Heidelberg, Germany, 2011.

8. De Roeck, D.; Slegers, K.; Criel, J.; Godon, M.; Claeys, L.; Kilpi, K.; Jacobs, A. I would DiYSE for it! A manifesto for do-it-yourself internet-of-things creation. In Proceedings of the 7th Nordic Conference on Human-Computer Interaction: Making Sense through Design, Copenhagen, Denmark, 4-17 October 2012; ACM: New York, NY, USA, 2012; pp. 170-179.

9. Ahmad, S.; Mehmood, F.; Mehmood, A.; Kim, D. Design and Implementation of Decoupled IoT Application Store: A Novel Prototype for Virtual Objects Sharing and Discovery. Electronics 2019, 8, 285. [CrossRef]

10. Espada, J.P.; Martínez, O.S.; Bustelo, B.C.P.G.; Lovelle, J.M.C. Virtual objects on the internet of things. IJIMAI 2011, 1, 23-29. [CrossRef]

11. Naeem, M.; Heckel, R.; Orejas, F. Semi-automated service composition using visual contracts. In Proceedings of the 7th International Conference on Frontiers of Information Technology (FIT '09), Abbottabad, Pakistan, 16-18 December 2009. Available online: https://www.researchgate.net/profile/Reiko_Heckel/publication/220791528_ Semi-automated_service_composition_using_visual_contracts/links/0deec52344956577d8000000.pdf (accessed on 1 October 2019).

12. Sirin, E.; Hendler, J.; Parsia, B. Semi-automatic composition of web services using semantic descriptions. In Proceedings of the 1st Workshop on Web Services: Modeling, Architecture and Infrastructure, Angers, France, 22 April 2003; pp. 17-24. 
13. Li, G.; Deng, S.; Xia, H.; Lin, C. Automatic service composition based on process ontology. In Proceedings of the IEEE Third International Conference on Next Generation Web Services Practices (NWeSP'07), Seoul, Korea, 29 October 2007; pp. 3-6.

14. Liu, L.; Liu, X.; Li, X. Cloud-based service composition architecture for internet of things. In Internet of Things; Springer: Berlin, Germany, 2012; pp. 559-564.

15. Urbieta, A.; González-Beltrán, A.; Mokhtar, S.B.; Hossain, M.A.; Capra, L. Adaptive and context-aware service composition for IoT-based smart cities. Futur. Gener. Comput. Syst. 2017, 76, 262-274. [CrossRef]

16. Ullah, I.; Sohail Khan, M.; Kim, D. IoT Services and Virtual Objects Management in Hyperconnected Things Network. Mob. Inf. Syst. 2018, 2018, 2516972. [CrossRef]

17. Hang, L.; Jin, W.; Yoon, H.; Hong, Y.; Kim, D. Design and Implementation of a Sensor-Cloud Platform for Physical Sensor Management on CoT Environments. Electronics 2018, 7, 140. [CrossRef]

18. Ahmad, S.; Hang, L.; Kim, D.H. Design and Implementation of Cloud-Centric Configuration Repository for DIY IoT Applications. Sensors 2018, 18, 474. [CrossRef] [PubMed]

19. Gama, K.; Touseau, L.; Donsez, D. Combining heterogeneous service technologies for building an Internet of Things middleware. Comput. Commun. 2012, 35, 405-417. [CrossRef]

20. Atzori, L.; Iera, A.; Morabito, G. From "smart objects" to "social objects": The next evolutionary step of the internet of things. IEEE Commun. Mag. 2014, 52, 97-105. [CrossRef]

21. Xiao, G.; Guo, J.; Da Xu, L.; Gong, Z. User interoperability with heterogeneous IoT devices through transformation. IEEE Trans. Ind. Inform. 2014, 10, 1486-1496. [CrossRef]

22. Perera, C.; Liu, C.H.; Jayawardena, S. The emerging internet of things marketplace from an industrial perspective: A survey. IEEE Trans. Emerg. Top. Comput. 2015, 3, 585-598. [CrossRef]

23. Schmid, S.; Bröring, A.; Kramer, D.; Käbisch, S.; Zappa, A.; Lorenz, M.; Wang, Y.; Rausch, A.; Gioppo, L. An architecture for interoperable IoT Ecosystems. In International Workshop on Interoperability and Open-Source Solutions; Springer: Cham, Germany, 2016; pp. 39-55.

24. Bröring, A.; Schmid, S.; Schindhelm, C.K.; Khelil, A.; Käbisch, S.; Kramer, D.; Le Phuoc, D.; Mitic, J.; Anicic, D.; Teniente, E. Enabling IoT ecosystems through platform interoperability. IEEE Softw. 2017, 34, 54-61. [CrossRef]

25. Krishnamachari, B.; Power, J.; Kim, S.H.; Shahabi, C. I3: An IoT marketplace for smart communities. In Proceedings of the 16th Annual International Conference on Mobile Systems, Applications, and Services, Munich, Germany, 10-15 June 2018; pp. 498-499.

26. Gil, D.; Ferrández, A.; Mora-Mora, H.; Peral, J. Internet of things: A review of surveys based on context aware intelligent services. Sensors 2016, 16, 1069. [CrossRef] [PubMed]

27. Saied, Y.B.; Olivereau, A.; Zeghlache, D.; Laurent, M. Trust management system design for the Internet of Things: A context-aware and multi-service approach. Comput. Secur. 2013, 39, 351-365. [CrossRef]

28. Kang, B.; Park, S.; Lee, T.; Park, S. IoT-based monitoring system using tri-level context making model for smart home services. In Proceedings of the 2015 IEEE International Conference on Consumer Electronics (ICCE), Las Vegas, NV, USA, 9-12 January 2015; pp. 198-199.

29. Sharma, S.; Shuman, M.A.R.; Goel, A.; Aggarwal, A.; Gupta, B.; Glickfield, S.; Guedalia, I.D.; Qualcomm Inc. Context Aware Actions among Heterogeneous Internet of Things (IOT) Devices. U.S. Patent 9,413,827, 9 August 2016.

30. Gochhayat, S.P.; Kaliyar, P.; Conti, M.; Tiwari, P.; Prasath, V.B.S.; Gupta, D.; Khanna, A. LISA: Lightweight context-aware IoT service architecture. J. Clean. Prod. 2019, 212, 1345-1356. [CrossRef]

31. Lee, T.D.; Lee, B.M.; Noh, W. Hierarchical cloud computing architecture for context-aware IoT services. IEEE Trans. Consum. Electron. 2018, 64, 222-230. [CrossRef]

(C) 2019 by the authors. Licensee MDPI, Basel, Switzerland. This article is an open access article distributed under the terms and conditions of the Creative Commons Attribution (CC BY) license (http://creativecommons.org/licenses/by/4.0/). 